\title{
On smooth integers in short intervals under the Riemann Hypothesis
}

\author{
by
}

Ti ZuO XuAN (Beijing)

1. Introduction. We say a natural number $n$ is $y$-smooth if every prime factor $p$ of $n$ satisfies $p \leq y$. Let $\Psi(x, y)$ denote the number of $y$-smooth integers up to $x$. The function $\Psi(x, y)$ is of great interest in number theory and has been studied by many researchers.

Let $\Psi(x, z, y)=\Psi(x+z, y)-\Psi(x, y)$. In this paper, we will give an estimate for $\Psi(x, z, y)$ under the Riemann Hypothesis $(\mathrm{RH})$.

Various estimates for $\Psi(x, z, y)$ have been given by several authors. (See [1]-[9].)

In 1987, Balog [1] showed that for any $\varepsilon>0$ and $X \geq X_{0}(\varepsilon)$ the interval $\left(X, X+X^{1 / 2+\varepsilon}\right]$ contains an integer having no prime factors exceeding $X^{\varepsilon}$.

Harman [6] improved this result, and he proved that the bound $X^{\varepsilon}$ on the size of the prime factors can be replaced by $\exp \left\{(\log x)^{2 / 3+\varepsilon}\right\}$.

Recently, Friedlander and Granville [3] improved the "almost all" results of Hildebrand and Tenenbaum [9] and proved the following result:

Fix $\varepsilon>0$. The estimate

$$
\Psi(x, z, y)=\frac{z}{x} \Psi(x, y)\left(1+O\left(\frac{(\log \log y)^{2}}{\log y}\right)\right)
$$

holds uniformly for

$$
x \geq y \geq \exp \left\{(\log x)^{5 / 6+\varepsilon}\right\}
$$

with

$$
x \geq z \geq x^{1 / 2} y^{2} \exp \left\{(\log x)^{1 / 6}\right\} .
$$

The authors of [3] also point out that up to now there is no indication of how to break the " $\sqrt{x}$ barrier", that is, to prove that $\Psi(x+\sqrt{x}, y)-$

1991 Mathematics Subject Classification: Primary 11N25.

Project supported by the National Natural Science Foundation of People's Republic of China. 
$\Psi(x, y)>0$ when $y$ is an arbitrarily small power of $x$; this is evidently the most challenging open problem in this area.

The problem is very difficult indeed. In this paper, we only prove that

$$
\Psi\left(X+\sqrt{X}(\log X)^{1+\varepsilon}, X^{\delta}\right)-\Psi\left(X, X^{\delta}\right)>0,
$$

even if the RH is true, and we state it formally as a theorem.

Theorem. If the $R H$ is true, then for any $\varepsilon>0, \delta>0$ and $X \geq$ $X_{0}(\varepsilon, \delta)$, the interval $(X, X+Y]$, where $\sqrt{X}(\log X)^{1+\varepsilon} \leq Y \leq X$, contains an integer having no prime factors exceeding $X^{\delta}$.

2. Proof of the Theorem. To prove the Theorem, we need the following lemmas.

LEmma 1. For $N, T \geq 1$ and any sequence $b_{n}$ of complex numbers, we have

$$
\int_{0}^{T}\left|\sum_{n \leq N} b_{n} n^{i t}\right|^{2} d t \ll(T+N) \sum_{n \leq N}\left|b_{n}\right|^{2} .
$$

Proof. See Theorem 6.1 of [10].

Lemma 2. If the $R H$ is true then for $1 / 2+\varepsilon \leq \sigma \leq 2$ we have uniformly

$$
\frac{\zeta^{\prime}}{\zeta}(s) \ll \log (|t|+2) .
$$

Proof. See [12, p. 340].

Let $0<\varepsilon<1 / 8$ be fixed. We put

$$
\begin{aligned}
& M=X^{1 / 2}(\log X)^{-1-\varepsilon}, \quad \quad N=(\log X)^{2+2 \varepsilon}, \\
& Y \geq \frac{X}{M}=X^{1 / 2}(\log X)^{1+\varepsilon}, \quad y=X^{\delta}, \\
& a(m)= \begin{cases}1 & \text { if } p \mid n \Rightarrow p \leq y, \quad M(s)=\sum_{M<m \leq 2 M} \frac{a(m)}{m^{s}} \text { otherwise, }\end{cases}
\end{aligned}
$$

As in [6] we will show that

$$
\int_{X}^{X+Y}\left(\sum^{*} a\left(m_{1}\right) a\left(m_{2}\right) \Lambda(r)\right) d x=Y^{2} M^{2}(1)+O\left(Y^{2}(\log X)^{-\varepsilon / 4}\right),
$$

where $*$ represents the summation conditions

$$
\begin{gathered}
m_{1} m_{2} r \in(x, x+Y], \quad X \leq x \leq X+Y, \\
M<m_{i} \leq 2 M, \quad i=1,2 .
\end{gathered}
$$



$\notin \mathbb{Z}$

By the Perron formula (see Lemma 3.19 of [12]) we have for $x \notin \mathbb{Z}, x+Y$

$$
\begin{aligned}
& \sum^{*} a\left(m_{1}\right) a\left(m_{2}\right) \Lambda(r) \\
& =\frac{-1}{2 \pi i} \int_{c-i T}^{c+i T} \frac{\zeta^{\prime}}{\zeta}(s) M^{2}(s) \frac{(x+Y)^{s}-x^{s}}{s} d s+O\left(\frac{x \log ^{2} x}{T}\right)+O(\log x),
\end{aligned}
$$

where $c=1+1 / \log X, T=X^{4}$, and the $O$ constants are absolute.

We now integrate (2.2) with respect to $x$ between $X$ and $X+Y$, and obtain that

$$
\begin{aligned}
& \int_{X}^{X+Y}\left(\sum^{*} a\left(m_{1}\right) a\left(m_{2}\right) \Lambda(r)\right) d x \\
= & \frac{-1}{2 \pi i} \int_{c-i T}^{c+i T} \frac{\zeta^{\prime}}{\zeta}(s) M^{2}(s) A(s) d s+O\left(\frac{X Y \log ^{2} X}{T}\right)+O(Y \log X),
\end{aligned}
$$

where

$$
A(s)=\frac{(X+2 Y)^{s+1}-2(X+Y)^{s+1}+X^{s+1}}{s(s+1)} .
$$

We note that $A(1)=Y^{2}$, and

$$
A(s) \ll \min \left(Y^{2} X^{\sigma-1}, X^{\sigma+1}|t|^{-2}\right) .
$$

From the definitions of $T$ and $Y$, it follows that the two error terms in (2.3) are $\ll Y^{2} \exp \left\{-(\log X)^{1 / 2}\right\}$.

By the theorem of residues, the integral on the right side of (2.3) is

$$
Y^{2} M^{2}(1)+\frac{1}{2 \pi i}\left(\int_{c-i T}^{\eta-i T}+\int_{\eta-i T}^{\eta+i T}+\int_{\eta+i T}^{c+i T}\right),
$$

where $\eta=1 / 2+\varepsilon / 3$.

Here we estimate $|M(s)|$ trivially as

$$
|M(s)| \leq M^{1-\sigma} .
$$

From this, (2.4) and Lemma 2, the integrals along the lines $[c-i T, \eta-i T]$ and $[\eta+i T, c+i T]$ are

$$
\begin{aligned}
& \ll \int_{\eta}^{c} M^{2-2 \sigma} X^{\sigma+1} T^{-2} \log T d \sigma \\
& \ll X^{2} T^{-2} \log T \ll Y^{2} \exp \left\{-(\log X)^{1 / 2}\right\} .
\end{aligned}
$$


Also,

$$
\begin{aligned}
\int_{\eta-i T}^{\eta+i T} \frac{\zeta^{\prime}}{\zeta}(s) M^{2}(s) A(s) d s \ll & Y^{2} X^{\eta-1} \log X \int_{0}^{X / Y}|M(\eta+i t)|^{2} d t \\
& +X^{\eta+1} \log X \int_{X / Y}^{T}|M(\eta+i t)|^{2} t^{-2} d t \\
= & I_{1}+I_{2} .
\end{aligned}
$$

By Lemma 1, we have

$$
\begin{aligned}
I_{1} & \ll Y^{2} X^{\eta-1} \log X\left(\frac{X}{Y}+M\right) M^{1-2 \eta} \\
& \ll Y^{2} \log X \cdot N^{-1+\eta} \ll Y^{2}(\log X)^{-\varepsilon / 4} .
\end{aligned}
$$

From Lemma 1 and (2.6) together with integration by parts we have

$$
\begin{aligned}
I_{2} & \ll X^{\eta+1} \log X\left(\frac{X}{Y}\right)^{-2}\left(\frac{X}{Y}+M\right) M^{1-2 \eta} \\
& \ll Y^{2} \log X \cdot N^{-1+\eta} \ll Y^{2}(\log X)^{-\varepsilon / 4} .
\end{aligned}
$$

So from (2.3), (2.5) and (2.7)-(2.10) we get (2.1). By Theorem 1 of [7], we have

$$
M(1)=\sum_{M<m \leq 2 M} \frac{a(m)}{m} \gg_{\delta} 1 .
$$

The Theorem follows from (2.1) and the above estimate.

REMARKS. Using the methods of this paper, we can prove the following results.

For any $X \geq X_{0}(\varepsilon)$, the interval $(X, X+Y]$ contains an integer having no prime factors exceeding $y$, where

(i) $X \geq Y \geq X^{1 / 2} \exp \left\{(\log X)^{5 / 6+\varepsilon}\right\}$ and $X \geq y \geq \exp \left\{(\log X)^{5 / 6+\varepsilon}\right\}$,

or

(ii) $X \geq Y \geq X^{1 / 2} \exp \left\{\frac{\log X}{(\log \log X)^{b}}\right\}$ and

$$
X \geq y \geq \exp \left\{C(\log X)^{2 / 3}(\log \log X)^{4 / 3+b}\right\},
$$

where $b$ is any fixed positive number and $C$ is a sufficiently large absolute constant.

The result suitable for the ranges (ii) is stronger than one of Harman [6] and the ranges (i) are wider than the ranges (1.2) and (1.3) of the asymptotic estimate of Friedlander and Granville [3] since the bound for $Y$ in (i) is independent of $y$. 
The proofs of the results are similar to that of the Theorem, but for the ranges (i) with

$$
M=X^{1 / 2} \exp \left\{(-\log X)^{5 / 6+\varepsilon}\right\}, \quad N=\exp \left\{2(\log X)^{5 / 6+\varepsilon}\right\},
$$

and

$$
\eta=1-\frac{c_{1}}{(\log X)^{2 / 3+\varepsilon}}
$$

and for the ranges (ii) with

$$
M=X^{1 / 2} \exp \left\{-\frac{\log X}{(\log \log X)^{b}}\right\}, \quad N=\exp \left\{\frac{2 \log X}{(\log \log X)^{b}}\right\},
$$

and

$$
\eta=1-\frac{c_{1}}{(\log X)^{2 / 3}(\log \log X)^{1 / 3}} .
$$

Moreover, in the proof we also need the following result: the estimate

$$
\frac{\zeta^{\prime}}{\zeta}(s) \ll \log (|t|+2)
$$

holds uniformly in the ranges $\sigma \geq 1-c_{1} /\left((\log X)^{2 / 3}(\log \log X)^{1 / 3}\right)$ and $|t| \leq X^{4}$. This estimate follows from an estimate of [11] and Theorems 3.10 and 3.11 of [12] with $\varphi(t)=\frac{302}{3} \log \log t$ and $\theta(t)=(\log \log t)^{2 / 3} /(\log t)^{2 / 3}$.

Acknowledgements. I would like to thank the referee for his comments and suggestions.

\section{References}

[1] A. Balog, On the distribution of integers having no large prime factors, Astérisque 147-148 (1987), 27-31.

[2] A. Balog and A. Sárközy, On sums of integers having small prime factors: II, Studia Sci. Math. Hungar. 19 (1984), 81-88.

[3] J. B. Friedlander and A. Granville, Smoothing "smooth" numbers, Philos. Trans. Roy. Soc. London Ser. A 345 (1993), 339-347.

[4] J. B. Friedlander and J. C. Lagarias, On the distribution in short intervals of integers having no large prime factors, J. Number Theory 25 (1987), 249-273.

[5] A. Granville, Integers, without large prime factors, in arithmetic progressions. II, Philos. Trans. Roy. Soc. London Ser. A 345 (1993), 349-362.

[6] G. Harman, Short intervals containing numbers without large prime factors, Math. Proc. Cambridge Philos. Soc. 109 (1991), 1-5.

[7] A. Hildebrand, On the number of positive integers $\leq x$ and free of prime factors $>y$, J. Number Theory 22 (1986), 289-307.

[8] A. Hildebrand and G. Tenenbaum, On integers free of large prime factors, Trans. Amer. Math. Soc. 296 (1986), 265-290.

[9] —, - Integers without large prime factors, J. Théor. Nombres Bordeaux 5 (1993), 411-484.

[10] H. L. Montgomery, Topics in Multiplicative Number Theory, Springer, 1971. 
[11] H. E. Richert, Zur Abschätzung der Riemannschen Zetafunktion in der Nähe der Vertikalen $\sigma=1$, Math. Ann. 169 (1967), 97-101.

[12] E. C. Titchmarsh, The Theory of the Riemann Zeta Function, 2nd ed., revised by D. R. Heath-Brown, Oxford Univ. Press, 1986.

Department of Mathematics

Beijing Normal University

Beijing 100875

People's Republic of China 